\title{
Saúde Baseada em Evidências e pandemias: dilemas sobre a Saúde Planetária e a decisão individual de impacto populacional
}

\author{
Evidence-Based Health and pandemics: Planetary Health dilemmas and \\ the individual decision of population impact
}

\section{Salud Basada en Evidencias y pandemias: dilemas de Salud Planetaria y la decisión individual de impacto poblacional}

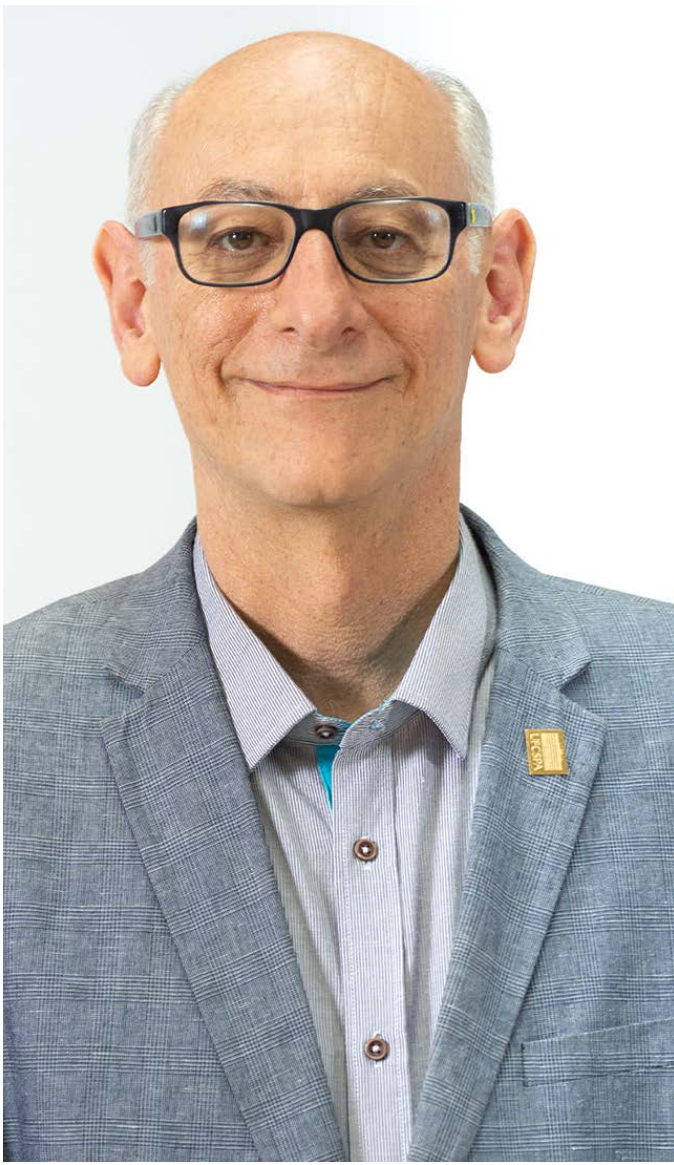

Durante a trajetória na prática clínica e na pesquisa, o médico, professor e pesquisador Airton Tetelbom Stein compreendeu que para ser um bom médico é necessário um embasamento científico aprofundado. Em entrevista à Reciis, Stein discute sobre o conceito de Saúde Baseada em Evidências (SBE) na sua relevância de integrar as melhores evidências com a experiência clínica e os valores e as preferências do paciente. A partir da sua experiência na Medicina de Família e Comunidade (MFC), Stein enfatiza que epidemias e pandemias, como a da covid-19, revelam o impacto das mudanças climáticas na saúde da população. Ressalta que um dos pilares da Atenção Primária à Saúde (APS) é o entendimento sobre as causas do surgimento das doenças no âmbito populacional. Nesse sentido, nas dinâmicas sociais, sobretudo as midiáticas acerca da covid-19, o pesquisador esclarece que as decisões sobre o tratamento precoce, sobre tomar ou não a vacina, dizem respeito à valorização de pressupostos baseados em informações não sistematizadas em detrimento de metodologias robustas, referem-se às decisões individuais que impactam na saúde da população. "As pessoas que têm se negado a realizar a vacinação precisam entender que isso não é apenas uma liberdade individual”. Airton Tetelbom Stein é professor titular da Universidade Federal de Ciências da Saúde de Porto Alegre (UFCSPA) e médico da família e comunidade do Grupo Hospitalar Conceição.

Palavras-chave: Saúde Baseada em Evidências; Saúde Planetária; Atenção Primária à Saúde; Pandemias; Informação. 


\section{ABSTRACT}

During his trajectory in clinical practice and in research, the physician, professor and researcher Airton Tetelbom Stein understood that, in order to be a good doctor, a deep scientific foundation is necessary. In an interview with Reciis, Stein discusses the concept of Evidence-Based Health in its relevance to integrate the best evidence with clinical experience and patients' values and preferences. Based on his experience in Family Practice, Stein emphasizes that epidemics and pandemics, such as covid-19, reveal the impact of climate change on the population's health. He also emphasizes that one of the pillars of Primary Health Care is the understanding of the causes of the emergence of diseases within the population. In this sense, in social dynamics, especially the mediatic dynamics about covid-19, Stein clarifies that decisions about early treatment, whether to vaccinate or not, concern the valuation of assumptions based on non-systematized information to the detriment of robust methodologies, refer to individual decisions that impact the health of the population. "People who have refused to vaccinate need to understand that this is not just a matter of individual freedom." Airton Tetelbom Stein is full professor of Public Health at the Federal University of Health Sciences of Porto Alegre (UFCSPA) and a family practice physician at Conceição Hospital Group.

Keywords: Evidence-Based Health; Planetary Health; Primary Health Care; Pandemics; Information.

\section{RESUMEN}

Durante su trayectoria en la práctica clínica y la investigación, el médico, profesor e investigador Airton Tetelbom Stein comprendió que, para ser un buen médico, es necesaria una base científica profunda. En entrevista a Reciis, Stein analiza el concepto de Salud Basada en Evidencias (SBE) en su relevancia para integrar la mejor evidencia con la experiencia clínica y los valores y preferencias del paciente. Basado en su experiencia en Medicina Familiar y Comunitaria (MFC), Stein enfatiza que las epidemias y pandemias, como covid-19, revelan el impacto del cambio climático en la salud de la población. Destaca que uno de los pilares de la Atención Primaria de Salud (APS) es la comprensión de las causas de la aparición de enfermedades en el ámbito poblacional. En este sentido, en dinámica social, especialmente en los medios de comunicación sobre covid-19, Stein aclara que las decisiones sobre el tratamiento temprano, la decisión de vacunarse o no, tienen que ver con la valoración de supuestos basados en información no sistematizada en detrimento de metodologías robustas, se refieren a decisiones individuales que impactan en la salud de la población. "Las personas que se han negado a vacinarse deben comprender que esto no es solo una cuestión de libertad individual”. Airton Tetelbom Stein es profesor de salud colectiva en la Universidad Federal de Ciencias de la Salud de Porto Alegre (UFCSPA) y médico de familia y comunidad en Grupo Hospitalar Conceição.

Palabras clave: Salud Basada en Evidencias; Salud Planetaria; Atención Primaria de Salud; Pandemias; Información. 


\section{Reciis: Poderia nos contar sobre a sua trajetória profissional e acadêmica na medicina e como a Saúde Baseada em Evidências (SBE) surge como um campo de interesse?}

Airton Stein: Eu me formei na Faculdade de Medicina da PUCRS, em 1981, e tive a oportunidade de realizar todo o meu último ano na Universidade de Miami, no Hospital Jackson Memorial. Nesse período foram vários os aprendizados: inicialmente tive contato com a Liga de Medicina de Família, que, no Brasil, em 1980, era uma especialidade muito pouco conhecida. Eu percebi que para ser um bom médico, além da prática clínica, era necessário ter um embasamento científico aprofundado e entender o contexto. Um curso que foi muito importante nesse momento da minha formação na Universidade de Miami foi o de abordagem de mecanismo de doença - no qual percebi a importância de integrar conhecimentos de fisiologia, patologia e epidemiologia com disciplinas clínicas. Essa experiência acadêmica levou-me a perceber que não se deve discriminar disciplinas básicas e de aplicação clínica, que valorizam o aprendizado baseado em problemas e no entendimento dos mecanismos de doenças.

Ao retornar para o Brasil, decidi que queria ser Médico de Família e Comunidade (MFC), que na época era chamado de medicina geral e comunitária. Fiz a residência na Secretaria de Saúde do Estado do Rio Grande do Sul, que era conhecida como residência do Murialdo, entre 1982 e 1983, quando tive a oportunidade de conhecer o professor Ellis Busnello, pioneiro em saúde comunitária e que anos depois foi o meu orientador de doutorado. Nesses dois anos de residência houve muitas mudanças na minha vida pessoal e profissional, conheci a minha esposa, Magda Costa, que também estava fazendo a formação em Medicina de Família e Comunidade e que nesses quase 40 anos compartilha comigo essa trajetória. O Murialdo foi um local de muito aprendizado sobre a importância de prevenção, rastreamento, utilização de ferramentas efetivas para o atendimento individual e de ações numa coletividade, na busca de intervenções centradas na pessoa. Percebi a necessidade de expandir e aperfeiçoar ações estratégicas para o controle da doença, entre as quais a detecção precoce, e de entender que, ao atender os pacientes no nível de atenção primária, os pacientes apresentam um espectro de não diferenciação.

Uma experiência marcante na residência foi iniciar uma pesquisa de motivos de consultas com os professores Bruce Duncan e Maria Inês Schmidt. Essa investigação originou a formatação do livro Medicina ambulatorial (DUNCAN et al., 2014), no qual elaborei dois capítulos 'Exame periódico de saúde' e 'Dispepsia'. Venho participando da elaboração das novas edições e, inclusive, da $5^{\mathrm{a}}$ edição, que está com perspectiva de ser lançada em breve.

Ao concluir a residência, a expectativa era ser contratado pela Secretaria de Saúde, já que até o ano anterior isso era garantido aos egressos da residência. No entanto, para esse grupo de residentes isso não se concretizou. Em 1984, o Grupo Hospitalar Conceição (GHC) abriu um processo seletivo, sob a coordenação do dr. Carlos Grossman, para a abertura de uma atividade inovadora em atenção primária no país - a criação da primeira Unidade de Medicina de Família, com a contratação de 8 MFCs -, no qual fui um dos selecionados.

Alguns anos depois, o serviço do GHC passa a ser denominado Serviço de Saúde Comunitária (SSC) e até hoje é um local de referência na assistência à população da zona norte de Porto Alegre e na formação de recursos humanos em atenção primária para o estado do Rio Grande do Sul - como uma estratégia para incentivar a formação desse profissional em todo o país. Participei do lançamento do Programa Saúde da Família pelo Ministério da Saúde, que depois o modificou para Estratégia Saúde da Família (ESF). Meu envolvimento foi muito ativo nesse processo, que também contou com a participação de colegas do Reino Unido, dos quais destaco os professores Andy Haines e Cecil Helman, que auxiliaram na definição do modelo de atendimento do SSC do GHC, assim como na implementação da atenção primária no país. 
Coordenei um encontro internacional, que foi muito importante para a discussão de estratégias para a implementação da Atenção Primária à Saúde (APS) no país, com uma organização não governamental chamada Action in International Medicine (AIM), evento que ocorreu em Porto Alegre, em 1993. Foi uma longa trajetória, na qual se valorizou cada vez mais um modelo de APS efetivo. Lembro que isso fez com que eu participasse como diretor de relações públicas da Associação Médica do Rio Grande do Sul (AMRIGS), em que destaco a coordenação das feiras de saúde que ocorriam em conjunto com os congressos dessa associação em cidades do interior do Rio Grande do Sul com estudantes de medicina, valorizando a prevenção.

No SSC do GHC, uma experiência que marcou a minha trajetória foi o convívio com a consultoria de epidemiologia dos professores Cesar Victora e Fernando Barros, que auxiliaram na organização dos serviços por meio de pesquisas operacionais. Esse contato estimulou que eu procurasse uma formação acadêmica, mestrado e doutorado, em epidemiologia. Realizei o primeiro mestrado no curso de Ciências Médicas da UFRGS, concluído em 1989 - o trabalho do mestrado foi Diagnóstico de saúde de uma comunidade na zona norte de Porto Alegre, RS: comparação entre os respondentes e os não respondentes (STEIN, 1989). Em 1989-1990 realizei um segundo mestrado, na London School of Hygiene \& Tropical Medicine, em Community Health for Developing Countries. O trabalho da dissertação foi A Cross-sectional study of Social Support and Hypertension in Porto Alegre, 1986 (STEIN, 1990). Na sequência, realizei o doutorado no curso de Ciências Médicas da UFRGS, que foi concluído em 1998 e cujo tema da tese foi Acesso a atendimento médico continuado: uma estratégia para reduzir a utilização de consultas não urgentes em serviços de emergência (STEIN, 1998).

Em 1999 iniciei minha carreira acadêmica como professor titular em saúde coletiva na Universidade Federal de Ciências da Saúde de Porto Alegre (UFCSPA) e continuo atuando até hoje. Nessa nova função, eu tive a oportunidade de conhecer e trabalhar com o professor e escritor Moacyr Scliar.

Na minha experiência como Médico de Família e Comunidade eu percebia que, além da sobrecarga no atendimento, tinha o desafio de saber lidar com o volume de informações científicas publicadas todos os dias. Constatei que era impossível que um médico conseguisse ler todos os artigos publicados. Portanto, seria essencial identificar quais artigos eram de boa qualidade metodológica, assim como aqueles de maior relevância para a prática clínica, considerando, principalmente, que os médicos que atuam na APS têm um elevado grau de incerteza no início da evolução clínica.

A definição de SBE como o "uso consciencioso, explícito e criterioso da melhor evidência disponível durante a tomada de decisões nos cuidados individuais dos pacientes" (SACKETT et al., 1996) é muito útil nesse paradigma. A prática de SBE é representada por três pilares: melhor evidência disponível; contexto e circunstâncias clínicas; e valores e preferências dos pacientes.

$\mathrm{O}$ item de melhor evidência disponível refere-se a pesquisas clinicamente relevantes centradas no paciente. Normalmente, incluem pesquisas sobre acurácia e precisão diagnóstica (incluindo exame clínico), marcadores prognósticos e seu poder de predição, eficácia e segurança de um tratamento, reabilitação ou estratégias preventivas.

O médico, ao longo do seu treinamento, precisa desenvolver habilidades clínicas, assim como aprender com as experiências prévias para identificar em cada paciente a evolução clínica e o seu diagnóstico. É necessário avaliar os riscos individuais e benefícios de potenciais intervenções, exposições ou testes diagnósticos e os valores e as expectativas pessoais do paciente. Além disso, a experiência clínica é necessária para integrar a evidência com os valores do paciente e as circunstâncias. Sobre o contexto, precisamos considerar o ambiente no qual o paciente está inserido e que define a probabilidade de pré-teste para identificar a gravidade do problema de saúde (ambulatorial, unidade de urgência, hospital); as questões 
de disponibilidade e acesso à propedêutica e aos tratamentos; as questões burocráticas/legais; ou outras situações do cenário que possam afetar a tomada de decisão.

No trabalho do MFC, a SBE é uma ferramenta essencial, mas que deve ser associada a outras habilidades e técnicas clínicas - como raciocínio clínico, método clínico centrado na pessoa, habilidades de comunicação -, assim como ao saber como trabalhar numa equipe multidisciplinar.

\section{Reciis: Como o senhor avalia o papel da MFC em situações de epidemia e pandemia, como esta que estamos vivendo agora?}

Airton Stein: Inicialmente, gostaria de citar os trabalhos desenvolvidos por Barbara Starfield (STARFIELD, 2002) sobre o papel da APS nos sistemas de saúde e a cobertura universal da saúde. Suas pesquisas sobre equidade contribuíram para a elaboração de políticas de saúde fundamentadas em evidências. Starfield descreveu quatro atributos do atendimento primário: primeiro contato, continuidade do atendimento, integralidade e coordenação do cuidado; e três atributos derivados: centralidade familiar, competência cultural e orientação comunitária. Nesse momento em que percebemos o impacto das mudanças climáticas na saúde, a APS deve identificar as exigências do Antropoceno - a era geológica atual, conotando o enorme impacto das atividades humanas sobre a saúde humana e sobre o ecossistema. O novo campo da Saúde Planetária não só procura enfrentar esse desafio do século XXI, mas também restabelece a conexão perdida da humanidade com a saúde do planeta.

A atenção primária tem como escopo o entendimento sobre as causas das doenças, portanto o enfoque não é apenas no atendimento aos pacientes, mas também nos aspectos de saúde populacional. As características da atuação do Médico de Família e Comunidade devem ter o compromisso de incluir ações nas comunidades onde atua e sobre os sistemas ecológicos que sustentam a saúde da humanidade. Para incorporar esse novo papel, o médico que atua na APS deve considerar a adição de mais um atributo: Saúde Planetária (BARROS et. al., 2021).

O surgimento da covid-19 ocorre em função do tipo de exploração do mundo feito pelos humanos. Existe uma perspectiva de novas pandemias, em função da diminuição da biodiversidade e da mudança climática. O Médico de Família e Comunidade e a equipe de saúde estão muitos interessados na 'causa das causas da doença'. Não estão focados só no atendimento individual do paciente, mas também estão focados nos problemas da população. Nesse sentido, temos que identificar qual a cultura, quais as características daquela comunidade, e em termos até históricos, para entender como se chegou àquele momento com aquele perfil de saúde.

Vários estudos no Brasil mostram que as comunidades que têm um serviço de atenção básica bem estruturado são as que tiveram desempenho melhor, ou menos pior, diante da pandemia. Além dessa questão da Saúde Planetária, a equipe de saúde de APS deve desenvolver os conhecimentos de vigilância epidemiológica e definir a sua atuação local com conhecimentos sobre a curva epidemiológica da covid-19 para deliberar como trabalhar com essas questões. Assim, quanto mais conhecimento de epidemiologia, mais condições a equipe de saúde da atenção primária terá para desenvolver um trabalho que responda às necessidades daquela população. Os médicos e a equipe de saúde devem entender o contexto que cada comunidade vivencia, analisar os riscos, danos e benefícios de cada intervenção proposta de acordo com as circunstâncias. 
Essa demanda está cada vez maior, é uma síntese de evidências qualitativas. ${ }^{1}$ Existe uma tendência, principalmente com o grupo de ensino e pesquisa da Noruega, em que eles desenvolveram um método de fazer síntese de evidências qualitativas (TOMA et. al., 2019).

A equipe de saúde tem que ter habilidades não apenas em pesquisa quantitativa, que evidentemente é muito mais utilizada para a tomada de decisão, mas também de pesquisa qualitativa. Deve-se, cada vez mais, realizar uma abordagem prática para fortalecer os sistemas de saúde através de uma lente de 'pensamento sistêmico'. Os profissionais de saúde devem capacitar-se para elaborar explicações práticas para questões complexas, que vão desde a concepção de intervenções orientadas ao sistema até a avaliação de seus efeitos. Cada vez mais, nós todos profissionais de saúde, devemos direcionar os nossos esforços ao fortalecimento dos sistemas de saúde universais. O fortalecimento dos sistemas de saúde ajuda a entender não apenas o que funciona, mas para quem e sob quais circunstâncias.

Reciis: A pandemia de covid-19, e a corrida/busca por respostas rápidas para combater a doença, destacou a importância da pesquisa científica e a sua comunicação. Presenciamos uma produção científica acelerada e crescente sobre o tema, bem como uma grande midiatização de resultados de estudos científicos, por vezes, controversos. Como o senhor avalia a importância da SBE nesse contexto? Como o senhor avalia a importância dos profissionais de comunicação?

Airton Stein: O aspecto principal é a importância da comunicação. Muitas vezes, o cientista e os profissionais de saúde não têm habilitação para comunicar as informações em uma linguagem para o usuário. Acho que esse é o primeiro passo: como possibilitar que a comunicação seja mais efetiva. Procurar evitar o jargão científico - um dos aspectos mais citados para a dificuldade das pessoas. Eu contribuí muito nessa pandemia para jornais e portais de notícias como O Estado de S.Paulo, Folha de S. Paulo e G1 e nunca havia tido essa experiência de ter contato com um jornalista. Percebi que o mais importante, do ponto de vista do comunicador, é encontrar a fonte confiável. Muitas vezes, o que vemos na mídia é a apresentação de informações controversas, pois não buscam uma fonte confiável.

Destaco fontes de evidências robustas como COVID19 Recommendations (ECOVID-19 RECMAP, c2021) e COVID-END, da Universidade McMaster (COVID-19 EVIDENCE NETWORK TO SUPPORT DECISION-MAKING COVID-END, c2021), que formam uma rede que reúne mais de 50 dos principais grupos de desenvolvimento de síntese de evidência, avaliação de tecnologia e diretrizes em todo o mundo. Ela cobre todo o espectro da resposta pandêmica, desde medidas de saúde pública e gerenciamento clínico até arranjos de sistemas de saúde e respostas econômicas e sociais. Também cobre todo o espectro de contextos em que a resposta pandêmica está ocorrendo, incluindo países de baixa, média e alta renda. A UFCSPA traduziu alguns desses materiais para orientação, e o importante é saber se comunicar e buscar fontes de informação confiáveis.

Vou dar um exemplo de uma revisão sistemática que fiz com um grupo. No início de março de 2021, um estudo mostrava que a obesidade era um fator de risco para mortalidade pela covid-19. Isso principalmente em países com alta taxa de obesidade, como Brasil e Estados Unidos. Em países asiáticos, que não têm tanta frequência de obesos, a mortalidade é bem menor. Convidei um grupo de colegas para revisar esse assunto. Minha ideia inicial era fazer um editorial para que isso fosse um indicador de saúde, um indicador que mostrasse para a população que não bastavam ações de prevenção à covid-19, mas também se preocupar com um fator de risco para outras doenças, como a obesidade, até para melhorar o indicador

1 O grupo GRADE CERQual - Confidence in the Evidence from Reviews of Qualitative Research (https://www.cerqual.org/ home/) - tem por objetivo avaliar a confiança nas evidências a partir de revisões de pesquisas qualitativas. 
em termos de mortalidade. Vimos que já havia mais de 60 meta-análises sobre esse item e não valia a pena fazer mais uma. Avaliamos a qualidade metodológica desses estudos e qual a síntese da informação. Utilizamos instrumentos que avaliam a qualidade de uma revisão sistemática, o AMSTAR 2 (sigla em inglês para A MeaSurement Tool to Assess Systematic Reviews), que possibilita realizar a avaliação de revisões sistemáticas de estudos randomizados e não randomizados de intervenções de saúde, e o método GRADE² (GUYATT et al., 2011). Percebemos que, do ponto de vista metodológico, a grande maioria dos estudos não foi adequada, o que limitava a interpretação.

Em função da pressa de querer publicar também há o fast track das revistas científicas com temas relacionados à covid-19. Muitas vezes os estudos, do ponto de vista metodológico, têm limitação. Então, é necessário desenvolver a habilidade de análise crítica do conhecimento. Isso é importante para jornalistas, profissionais de saúde, gestores, mas também para a população em geral. Existe uma iniciativa que se chama Healthy Choices (Escolhas Saudáveis)3 , que pertence ao grupo no qual fiz meu pós-doutorado na Noruega ${ }^{4}$ Eles orientam alunos do ensino fundamental em Uganda a avaliar criticamente os pressupostos de intervenções, por exemplo: termos um tratamento novo, caro ou de marca, não significa que seja melhor ou mais seguro que outros tratamentos (THAT’S A CLAIM, c2021). O legado que temos de ter é que o aprendizado não é só ter acesso à informação, mas ter senso crítico sobre tudo que chega até nós. Precisamos de uma habilidade de avaliação crítica. Saber diferenciar fato e opinião é um atributo essencial de cidadania.

Numa aula sobre pesquisa qualitativa, uma professora ressaltou que, de acordo com a lente de cada um, haverá uma opinião diferente. Ela era inglesa e fez o comentário para exemplificar a partir de um jogo de futebol entre Inglaterra e Brasil. Se alguém perguntar como foi o jogo a um torcedor brasileiro e a outro inglês, cada um vai ter uma opinião diferente. Se perguntar a um jogador de cada time, também vai ser diferente. Se a pergunta for para um analista de tática de jogo, ele vai ter também outra visão. Então, isso é opinião, a percepção de acordo com a lente de cada um. No futebol, isso fica bem evidente. Nesse sentido, é preciso levar em conta a opinião, mas também os fatos, principalmente para a tomada de decisão.

Reciis: Ao longo da pandemia, vimos, no campo assistencial, o incentivo e o estabelecimento de protocolos terapêuticos pautados em estudos de baixa ou nenhuma evidência científica, fator que gerou grande embate entre cientistas e profissionais de saúde, levantando o questionamento sobre a importância da competência informacional desses atores para buscar as melhores evidências científicas para nortear as suas práticas. Como o senhor avalia a formação do profissional de saúde quanto à SBE na atualidade? Os profissionais da ponta estão preparados para basear a sua prática nas melhores evidências científicas?

Airton Stein: "The desire for action" foi um termo usado pela Margaret Thatcher, ex-primeiraministra do Reino Unido. Eu assisti a essa entrevista quando morava na Inglaterra. Foi no final da guerra entre Inglaterra e Argentina, em função das Ilhas Falkland (para ingleses) e Malvinas (para argentinos). A repórter perguntou por que ela, como comandante supremo, tinha mandado afundar o Belgrano, navio

2 O GRADE (Grading of Recommendations Assessment, Development and Evaluation) desenvolveu uma abordagem comum, sensata e transparente de qualidade de classificação (ou certeza) das evidências e da força das recomendações.

3 Healthy Choices - Escolhas Saudáveis (https://www.informedhealthchoices.org). Pessoas em muitos países e regiões que desenvolvem, avaliam ou contextualizam recursos de 'escolhas saudáveis informadas'.

4 O grupo Cochrane Effective Practice and Organisation of Care (EPOC) (https://epoc.cochrane.org/norwegian-satellite) - Prática Efetiva e Organização do Cuidado - conduz, apoia e publica revisões sistemáticas das evidências globais para orientar a tomada de decisões do sistema de saúde mundial a fim de melhorar os serviços de saúde e os resultados da saúde da população. 
argentino. Depois da guerra foi comentado que esse abalroamento e consequente afundamento do navio foi um problema ético. $\mathrm{O}$ ataque ocorreu fora da zona de exclusão estabelecida pelo governo britânico em torno das ilhas. Morreram 323 marinheiros argentinos, praticamente metade de todas as baixas argentinas durante o conflito. A resposta da Margaret Thatcher foi aquela esperada de um comandante em chefe: "Os nossos meninos estavam em risco, era uma área de conflito". Ao final da entrevista, a repórter usou esse termo - "the desire for action" (desejo de agir, em tradução livre). Geralmente, com relação ao médico, o desire for action é prescrever um medicamento, orientar uma conduta invasiva, e, muitas vezes, a conduta deveria ser expectante ou a de buscar mais informações.

Outro termo que destaco: 'one size fits all' (um tamanho serve para todos, em tradução livre, no sentido de que uma mesma abordagem é utilizada em todos os casos) é um problema muito frequente. Muitas vezes, há uma tendência tanto do profissional de saúde como do gestor de propor uma conduta padronizada, e não de acordo com a necessidade. 'One size fits all' é como se um tamanho de sapato coubesse em todos os pés, o que não é verdade. Para evitar isso, é preciso estar atento às características de cada comunidade, à história de vida de cada paciente para identificar a conduta customizada.

O 'Elephant in the room'é outro termo que uso e que responde em parte a essa pergunta. Muitas vezes as pessoas não discutem temas como a mudança climática, com ondas de calor excessivo e, agora, a crise hídrica no Brasil, as enchentes na Europa, e a migração que muitas vezes é decorrente da mudança climática, que gera instabilidade e conflitos, principalmente, com aqueles que trabalham no campo. Tudo isso em função da mudança climática. É a causa das causas. Então, acaba se discutindo cada um desses temas como se não houvesse vinculação com a mudança climática e uma reflexão em busca de uma economia verde: ter um senso virtuoso para se usar mais transporte ativo; incentivar o plantio de árvores nas cidades; economizar água; evitar gastos excessivos. Muitas vezes não se discute o aspecto principal. Há estudos que mostram até que a violência aumenta em função das ondas de calor, mostrando que existe a necessidade de desenvolver estratégias preventivas e coletivas para diminuir a iniquidade, e mostrando também que a solução é o engajamento de todos os atores da sociedade.

A violência e a iniquidade geram apartação social. Em setembro de 1993, fui ao lançamento do livro O que é apartação: o apartheid social no Brasil, de Cristovam Buarque (Coleção Primeiros Passos) da Editora Brasiliense. A definição de apartação social é a diferença que os brasileiros ricos e quase ricos começam a assumir em relação aos pobres; é a aceitação da miséria ao lado, com o cuidado de se construir mecanismos de separação. Cristovam Buarque escreveu uma dedicatória para a minha filha, que na época tinha 3 meses: "Espero que essas ideias ajudem a construir um Brasil onde a Shani não precise escolher em qual país viver, porque o Brasil será só um”. Infelizmente, o país aumentou a iniquidade, e com a pandemia da covid-19 ela se tornou mais evidente.

Em relação à assistência, os profissionais que atuam na APS devem estar preparados para basear sua prática nas melhores evidências científicas e, principalmente, no caso de elas não serem tão robustas, a melhor conduta de intervenção é promover um diálogo entre o profissional e o paciente sobre qual é o melhor caminho a seguir. Esse tema foi pauta do congresso da Cochrane, no Canadá, que mostrou a relevância da decisão compartilhada, usando como exemplo uma criança com diagnóstico de otite média aguda para auxiliar a família na decisão da indicação ou não do uso de antibiótico.

Destaco também a importância da síntese da evidência, a partir de perguntas bem formuladas e que reflitam as prioridades, assim como a história e a cultura da comunidade. As intervenções não apresentam o mesmo nível de efetividade e requerem consideração sobre riscos e benefícios, e também quanto a barreiras e facilitadores na implementação. Ultimamente, tem sido elaborada a síntese de evidências de uma forma mais rápida e a sua utilização sobre um determinado tema vem auxiliando na tomada de decisão. Um aspecto em que a Comissão Nacional de Incorporação de Tecnologias no Sistema Único de Saúde (Conitec) 
tem investido bastante é nos Protocolos Clínicos e Diretrizes Terapêuticas (PCDT). Essa orientação é muito útil para gestores e profissionais de saúde. No entanto, a implementação dessas diretrizes ainda é muito irregular - ela não é implementada na maior parte dos serviços de saúde da linha de frente. Há a necessidade de se fazer uma tradução dessas necessidades para cada circunstância. Uma iniciativa da Conitec é já disponibilizar essa informação e termos acessíveis para a população leiga. Esta é certamente uma estratégia dessa orientação: facilitar a tradução de uma informação robusta, do ponto de vista metodológico, para a prática clínica, e o importante é desenvolver estratégias que facilitem a sua implementação.

Reciis: Como podemos aproveitar as lições da pandemia da covid-19 para estabelecer estratégias que visem o uso da SBE como base para a tomada de decisão nos cuidados de saúde? Quais seriam as melhores estratégias para esse fim, na sua opinião?

Airton Stein: A principal estratégia é valorizar a Atenção Primária à Saúde em contraponto à valorização que é apresentada pela mídia, que se concentra em leitos hospitalares, leitos de UTI, atendimento dos casos mais graves e risco de morte. Evidente que todos nós nos preocupamos muito com os casos que evoluíram para o risco de morte, mas o foco principal tem que ser a valorização da atenção primária, onde ocorre a maior parte dos problemas com evolução para casos mais graves e que podem ser evitados. Assim, cito um guia de avaliação de tecnologia de saúde na atenção básica que foi elaborado pela Rede Brasileira de Avaliação de Tecnologias em Saúde (REBRATS) (BRASIL; HOSPITAL ALEMÃO OSWALDO CRUZ, 2017) e que poderia ser atualizado dentro desse contexto da covid-19, principalmente, com a capacidade para resolver a maior parte dos problemas de saúde da população. Na atenção primária há mais resolutividade, e, muitas vezes, quando pensamos em tecnologia, pensamos em equipamento de alta densidade tecnológica. Mas, veja por exemplo o caso do cinto de segurança, que a maioria das pessoas usa hoje em dia. Sabe-se que há uma multa para quem não usa e é muito difícil ver alguém andar de carro sem o cinto. Da mesma maneira, é difícil um motociclista não usar o capacete. Isso porque são contribuições tão evidentes que foram introjetadas pela população. Um aspecto que vai fazer parte da nossa rotina é o uso de máscara, pois é efetivo não apenas para evitar a transmissão da covid-19, mas todas as doenças infectorrespiratórias. O inconveniente é tão pequeno e o uso da máscara não causa qualquer prejuízo. Óbvio que há algum desconforto, mas o benefício é enorme no combate à transmissão da covid-19 e de outras doenças infecciosas respiratórias. Esse tipo de ação preventiva deve ser estimulado e deve ocorrer muito mais no nível da atenção primária. Nesse sentido, o Ministério da Saúde e os serviços de saúde devem desenvolver estratégias para enfatizar tecnologias que sejam utilizadas por mais pessoas e de forma cotidiana, próximas da vida delas, e respeitando a cultura local.

Reciis: Que avaliação o senhor faz do desenvolvimento científico na atualidade e qual sua perspectiva para os próximos anos?

Airton Stein: O mais importante são as Healthy Choices, que eu havia comentado. As pessoas podem fazer escolhas saudáveis. A partir dos dados coletados pelo Programa Nacional de Controle do Tabagismo (PNCT), coordenado pelo Instituto Nacional do Câncer (INCA), a redução do número de pessoas que buscaram tratamento durante o ano de 2020 foi de $66 \%$ em nível nacional em relação ao ano anterior (INCA, 2021). Nos próximos anos, precisaremos nos esforçar, como sociedade, para lidar com o impacto da pós-covid-19 de maneira direta e indireta. O enfoque da prevenção deve sempre ser muito valorizado. Infelizmente, as pessoas têm que avaliar criticamente os fatos, a partir de seus 
pressupostos, identificando o que funciona e o que não funciona. Qual é o benefício e qual é o dano? Qual é o custo? Tem que se levar em conta, principalmente, aqueles estudos com metodologias robustas e que têm relevância para a prática clínica.

Sempre há uma limitação de recursos, quando se vai decidir quais intervenções serão implementadas pelo serviço de saúde. A definição de prioridade deve ser feita por todos os atores da sociedade: gestores, profissionais de saúde, pessoas que vivem e atuam numa determinada comunidade. Deve-se partir do pressuposto de que essas pessoas estão preparadas para avaliar criticamente a definição dessas prioridades. Nesse sentido, há possíveis conflitos de interesse. Não se deve levar em conta apenas observações fortuitas, anedóticas, ou seja, informações não confiáveis para definir a interpretação dos dados.

Muitas vezes, opiniões de experts não são necessariamente fontes confiáveis. A revisão por pares e a publicação num jornal científico não garantem que as comparações sejam bem-feitas. É preciso se ter mais confiança na síntese das evidências com tudo que se publicou sobre determinado problema. Por isto as revisões sistemáticas e bem elaboradas são tão valorizadas: elas observam criticamente a metodologia e as escolhas, dependem do julgamento dos problemas da evidência para, ao final, avaliar os benefícios e danos daquela intervenção.

Numa banca de dissertação de mestrado na qual fui avaliador, a aluna relatou que, ao se identificar com os resultados da pesquisa, a atuação dela como nutricionista passou a fazer mais sentido, pois ela começou a ver o que funcionava e o que as pessoas entendiam ou não sobre a proposta que ela apresentava aos usuários. A pesquisa tem esse componente de avaliação crítica de tudo que acontece na vida das pessoas. É por isto que investir em pesquisa é tão importante: para termos opções mais efetivas e que atendam às necessidades da população.

\section{Reciis: Na sua opinião, qual será o impacto desse desenvolvimento no campo da SBE?}

Airton Stein: Penso que deverá modificar o modelo assistencial, do modelo de pagamento por procedimento (fee for service) para um modelo value in health, onde o 'valor' na assistência médica é derivado da medição dos resultados da saúde em relação ao custo da entrega dos resultados.

Em 30 de setembro de 2021 foi publicado um relatório, do qual participei como coautor, que analisa abordagens para a realização de cuidados de saúde baseados em valores centrados no paciente: "PersonCentred Value-Based Health Care (PCVBHC)". O documento foi publicado no site do projeto Sprink (https://www.sprink.co.uk/pcvbhc-report). O relatório tem como objetivo avaliar as oportunidades de desenvolvimento de sistemas de saúde que centrem nas preferências individuais dos pacientes, bem como na prestação de serviços de alta qualidade, ao mesmo tempo que permitem a gestão de orçamentos de saúde da população. O estudo partiu da seguinte temática: "Ao considerar a implementação de Saúde Baseada em Valor e Saúde Centrada na Pessoa percebe-se que há necessidade de sabermos sobre as preferências e os objetivos das pessoas ao receberem um atendimento nos serviços de saúde. Também é essencial identificar o custo do atendimento. $\mathrm{O}$ valor monetário dos atendimentos tem aumentado consideravelmente em todo o mundo, o que é uma barreira para a implementação".

Foi utilizada uma metodologia estruturada apoiada por uma revisão pragmática da literatura e por um painel consultivo. Como resultados, o relatório identificou que é essencial realizar a tomada de decisão compartilhada, considerando que os clínicos, assim como a pessoa que recebe o atendimento, devem atuar em conjunto para propor uma solução aos problemas apresentados. Segundo o documento, as pessoas que recebem o atendimento devem estar envolvidas em todo o processo e desenvolver estratégias com o intuito de implementar tecnologias efetivas ao longo da vida. O estudo conclui que, ao definir sobre 
as alocações de recursos, os sistemas de saúde devem ser transparentes sobre a metodologia que sustenta a tomada de decisão.

Então, no value in health (valor em saúde), em inglês há duas expressões: what matters? É a primeira pergunta que é feita ao paciente - "qual é o seu problema?". No entanto, o mais relevante é what is the matter with you? - "o que é importante para você?” Por isso que cada vez mais os desfechos incluídos em pesquisas científicas são definidos em conjunto por um grupo de pacientes. Não é apenas o pesquisador que vai definir o desfecho mais relevante - os valores e as preferências do paciente têm que ser levados em conta. O desenvolvimento da SBE são o share decision-making, decisão compartilhada com o paciente, e a identificação de desfechos que sejam relevantes para o paciente. Evidentemente que alguns desfechos são fáceis de identificar - evitar a morte é um desfecho importante. Com as tecnologias que temos em UTI pode-se prolongar a vida, mas sem qualidade. Isso é um questionamento ético, e a definição deve ser realizada em conjunto com o paciente e a família dele, e é essencial facilitar a transição da vida para a morte, com um cuidado paliativo de qualidade.

Nossa cultura não tem esse planejamento de "o que eu quero para minha vida, se tiver limitações de tomada de decisão?," ou mesmo "e se eu tiver diminuição de habilidade de fala?" ou "se eu tiver diminuição da capacidade de me alimentar sozinho"? "Se eu não conseguir desempenhar essas tarefas sem o apoio de outras pessoas?” Muitas vezes, essas perguntas são muito pessoais - como cada um vai aceitar esse momento de limitações em função de uma doença grave. Quanto mais preparadas as pessoas estiverem para definir isso, melhor vai ser a decisão baseada nos valores e nas preferências de cada um. Essa pandemia nos induziu a pensar e a conhecer mais de nós mesmos, em primeiro lugar. No que diz respeito aos nossos familiares, que eventualmente podem estar numa circunstância de risco de vida, é preciso saber qual a orientação em termos de cuidado que se deve seguir em função de limitações. A covid-19 atingiu pessoas que não tinham expectativa de morrer durante a pandemia, mas morreram. Vimos muita gente jovem e principalmente indivíduos com mais idade que não conseguiram se despedir dos seus familiares.

Reciis: Há uma questão que a pandemia coloca que é sobre a tomada de decisão. Algumas se expressam fortemente nas ações midiáticas: sobre a escolha da vacina - qual tomar, se toma ou não a vacina, ou se deve tomar determinado medicamento como prevenção. Como você avalia o compartilhamento de decisões entre médico e paciente diante dessas dinâmicas, nessa perspectiva midiática, que tem acontecido com relação à pandemia?

Airton Stein: Uma coisa é a vacina. Estamos lidando com uma doença infectocontagiosa. Então, se eu decido não me vacinar, não é apenas um impacto para a minha vida, essa decisão impacta uma sociedade. Esse é o grande problema. As pessoas que têm se negado a se vacinar precisam entender que isso não é apenas questão de liberdade individual. Isso tem impacto para o entorno no qual elas circulam. Isso é uma visão muito norte-americana, e da qual parte da nossa sociedade compartilha, ao valorizar muito a decisão individual.

No caso da vacina e do uso do cinto de segurança no carro, há muito mais benefícios do que danos, e essas intervenções são em nível populacional. O que vejo é que muitas pessoas agem procurando mais os benefícios individuais. Eu peguei uma época em que o cigarro já não era mais aceitável em ambiente internos, mas, se você ia entrar num avião e queria fumar, a recomendação era de fumar apenas nas últimas fileiras do avião. Observando em perspectiva, nota-se que era uma adaptação completamente inadequada. Fumar em qualquer lugar do avião acabava impactando a todos, como fumantes passivos. Então, vemos que a sociedade, por interesses comerciais e conflitos de interesses, demora um tempo a tomar medidas que serão entendidas como mais recomendáveis. Voltando ao cigarro, até pouco tempo era proibido veicular 
propaganda de cigarro em eventos esportivos. Agora isso já está voltando - conseguiram uma exceção para as corridas de Fórmula-1. Penso que a sociedade vai moldando a sua conduta em função de conhecimento. O conhecimento adquirido certamente é um aspecto a ser levado em conta, mas a sociedade se molda também sob pressão de determinados grupos. Muitas vezes, os interesses das indústrias acabam também fazendo parte da tomada de decisão sobre uma orientação.

Voltando à pergunta, muitos que são contra a vacina dizem que desconfiam porque ela foi criada muito rapidamente. No entanto, o que houve foi uma colaboração enorme da comunidade científica com métodos mais modernos que possibilitaram, em pouco tempo, que a gente pudesse ter uma vacina eficaz. Em relação ao desenvolvimento da SBE, um aspecto importante são as estratégias que possibilitam a internacionalização, para que cada vez mais haja a colaboração com instituições de outros países.

Há dois projetos de internacionalização com os quais estou envolvido, o primeiro na University of Texas MD Anderson Cancer Center, em parceria com a Universidade do Novo México (https://hsc.unm.edu/ echo), por meio do Projeto ECHO, sobre gravidez não planejada e auxiliando na infraestrutura da UFCSPA para que tenhamos um hub para a América Latina por meio do projeto. O outro projeto é o Sort-it, em que estamos desenvolvendo um curso de capacitação para profissionais de saúde e gestores que visa desenvolver pesquisas operacionais em relação ao uso inadequado de antimicrobianos. O projeto conta com a parceria da Organização Pan-Americana da Saúde (OPAS).

É preciso capacitar tanto os profissionais de saúde quanto a população para evitar o sobrediagnóstico (o termo em inglês é overdiagnosis). Minha expectativa, depois que nos liberarmos mais dessa pandemia, é que certamente haverá uma tendência de as pessoas quererem viajar mais, fazer mais festa, beber mais álcool e fazer coisas que hoje estão mais restritas. Sabemos que haverá mais riscos, e uma coisa que provavelmente vai ocorrer é um aumento na busca por consultas e exames complementares, que muitas vezes não são necessários. Há uma tendência ao sobrediagnóstico. É preciso ter como prioridade pensar na equidade e na sustentabilidade do sistema.

A prática clínica, muitas vezes, adota a polifarmácia muito mais em função da busca da solução de um sintoma do que em função do impacto que isso terá no desfecho de relevância clínica. Em conclusão, a pandemia nos trouxe grandes perdas e sofrimento, e a exacerbação da iniquidade na nossa sociedade; no entanto, também nos trouxe grandes avanços tecnológicos, como o ensino a distância, a telemedicina, o trabalho em home office e a saúde digital. Essas estratégias podem melhorar a qualidade de vida e diminuir a iniquidade.

\section{REFERÊNCIAS}

BARROS, Enrique Falceto de et al. Planetary health care and Barbara Starfield's legacy. BMJ GH Blogs, Londres, 10 jun. 2021. Disponível em: https://blogs.bmj.com/bmigh/2021/06/10/planetary-health-care/. Acesso em: 4 out. 2021

BRASIL. Ministério da Saúde; HOSPITAL ALEMÃO OSWALDO CRUZ. Guia de avaliação de tecnologias em saúde na Atenção Básica. Brasília, DF: Ministério da Saúde, 2017.

COVID-19 EVIDENCE NETWORK TO SUPPORT DECISION-MAKING (COVID-END). COVID-END. Hamilton: McMaster Health Forum, c2021. Disponível em: https://www.mcmasterforum.org/networks/covid-end. Acesso em: 24 set. 2021.

DUNCAN, Bruce B. et al. Medicina ambulatorial: condutas de atenção primária baseadas em evidências. 4. ed. Porto Alegre: Artmed, 2014.

ECOVID-19 RECMAP. COVID19 Recommendations and Gateway to Contextualization. [s. I.]: Cochrane Canada, c2021. Disponível em: https://covid19.recmap.org/. Acesso em: 24 set. 2021. 
GUYATT, Gordon et al. GRADE guidelines: 1. Introduction - GRADE evidence profiles and summary of findings tables. Journal of Clinical Epidemiology, Oxford, v. 64, n. 4, p. 383-394, jan. 2011. DOI: 10.1016/j. jclinepi.2010.04.026. Disponível em: https://www.jclinepi.com/article/S0895-4356(10)00330-6/fulltext. Acesso em: 4 out. 2021

INSTITUTO NACIONAL DO CÂNCER (INCA). Saúde desenvolve estratégias para conter queda em procura por tratamento contra o tabagismo na pandemia. Rio de Janeiro: Inca, 25 ago. 2021. Disponível em: https://www.inca.gov.br/noticias/saude-desenvolve-estrategias-para-conter-queda-em-procura-portratamento-contra-o-tabagismo. Acesso em: 06 out. 2021.

SACKETT, David L. et al. Evidence-based medicine: what it is and what it isn't. BMJ, Londres, v. 13, n. 312, p. 71-72, 1996. DOI: https://doi.org/10.1136/bmj.312.7023.71. Disponível em: https://www.bmj.com/ content/312/7023/71. Acesso em: 6 out. 2021.

STARFIELD, Bárbara. Atenção Primária: equilíbrio entre necessidades de saúde, serviços e tecnologia. Brasília, DF: Unesco: Ministério da Saúde, 2002. 726 p. Disponível: em: http://www.dominiopublico.gov.br/ pesquisa/DetalheObraForm.do?select_action=\&co obra=14609. Acesso em: 24 set. 2021.

STEIN, Airton. A cross-sectional study of social support and hypertension in Porto Alegre. 1990. Dissertação (Mestrado em Community Health For Developing Countries) - London School of Hygiene and Tropical Medicine, Londres, 1990.

STEIN, Airton. Acesso a atendimento médico continuado: uma estratégia para reduzir a utilização de consultas não urgentes em serviços de emergência. 1998. 248 f. Tese (Doutorado em Medicina: Ciências Médicas) - Faculdade de Medicina, Universidade Federal do Rio Grande do Sul, Porto Alegre, 1998. Disponível em: https://lume.ufrgs.br/handle/10183/1919. Acesso em: 24 set. 2021.

STEIN, Airton. Diagnóstico de saúde de uma comunidade na zona norte de Porto Alegre, RS: comparação entre os respondentes e os não respondentes. 1989. 113 f. Dissertação (Mestrado em Medicina: Ciências Médicas) - Faculdade de Medicina, Universidade Federal do Rio Grande do Sul, Porto Alegre, 1989. Disponível em: https://lume.ufrgs.br/handle/10183/1388. Acesso em: 24 set. 2021.

THAT'S A CLAIM. Based on faulty logic: "new is better!". Oslo: Centre for Informed Health Choices; Santiago de Chile: Epistemonikos Foundation, c2021. Disponível em: https://thatsaclaim.org/health-primary-school/ beware/new-is-betterl. Acesso em: 4 out. 2021.

TOMA, Tereza Setsuko; BARRETO, Jorge Otávio Maia; LEWIN, Simon (org.). GRADE-CERQual: uma abordagem para avaliar a confiança nos resultados de Sínteses de evidências qualitativas. Tradução: Kassia Fernandes de Carvalho. São Paulo: Instituto de Saúde, 2019. Disponível em: https://www.arca.fiocruz.br/ handle/icict/44095. Acesso em: 8 out. 2021. 